\title{
Caries Research
}

Acarbose 17

Acid production 17,83

- tolerance 83

Activity, caries 405

Adhesion of mutans streptococci 171

Adults 294

$\mathrm{AmF} / \mathrm{SnF}_{2} 462$

$\alpha$-Amylase 17

Antibiotic use 374

Antimicrobial activity 334

Approximal surfaces 36

Artificial carious enamel lesions 474

Australia, caries 366

Beverage 382

Bifidobacteria 308

Biofilm 97, 334

Biological markers 147

Biomechanics 70

Biomineralization 132

Bitewing radiography 36

Body mass index 468

Bovine enamel 261, 359

Calcium 269

- analysis 422

Candida 83

Caries 70,250

- arrestment 92

- control 387,391

- detection 36, 137, 314, 397

- diagnostics 339

- prevalence 436

- prevention 261

- removal 354

- trends 2

-, occlusal and approximal surfaces 442

Cariogenicity 83

Carryover effects 331

Casein 171

- phosphopeptide 430

Checkerboard analysis 462

Chewing gum 455

Children 382, 430, 436, 484

-, caries 366

Chlorhexidine 92, 331

Clinical trial 354, 430, 462

$\mathrm{CO}_{2}$ laser 261

Crossover studies 331

Cross-sectional hardness 474
Deciduous dentition 2

Demineralisation/Demineralization 50, 261, 278, 302

Demineralising solutions and gels 474

Dental care 294

- caries 43, 245, 286, 294, 302, 308, 331, $339,345,430$

- enamel 50,163

- erosion 126, 163, 449

- fillings 286

- fluorosis, prevalence 250

- plaque 64, 308

Dentifrice(s) 50, 57, 142, 278, 387, 391, 430

Dentin 70

Dentinal caries 92

- lesions 137

Dentine 119,415

- caries 314, 339

Detection, caries 405

DIAGNOdent 397

Diagnosis 245

Dicalcium phosphate dihydrate 254

Diet 331

dmfs index 155

DMFT index 468

Drinking water 147

Early childhood caries 25, 110, 155, 484

Enamel 10, 119, 132, 269, 415, 491

- caries 314

- hypoplasia 25, 345

Epidemiology 2, 245, 286, 436

Erosion 10, 57, 132, 269, 415

-, dentine 422

Floss 387

Fluoridated hydroxyapatite 132

Fluoride(s) 10, 50, 142, 147, 250, 269, 278, $331,366,387,391,430$

- dentifrice 64

- intake 382

- varnish 484

Fluorosis 147, 366

Food 382

Gap size 97

Glass ionomer 97
Hardness 359, 491

Human milk 171

Hydrofluoric acid 126, 449

Hydroxyapatite 254, 322

Hypohalous acid 334

ICDAS-II 405

In situ study 415

- vitro 359

Income inequality 294

Interdental sites 64

International Caries Detection and Assessment System 442

Jamaica 250

Lactobacilli 83

Laser fluorescence $36,137,302$

Logistic regression 468

Longitudinal microradiography 422

- study 286

Low fluoride concentration 278

Maltotriitol 17

Maturation 491

Mechanical properties 70

Microcomputed tomography 314

Microflora 354

Microtomography 314

Mouthrinse 387, 391

Mutans streptococci 455

Nails 142, 147

Nanoindentation 70

Nanostructure 132

Non-cavitated lesions 2

Nyvad's system 405

Occlusal caries 137

- surfaces 405

Octacalcium phosphate 322

Optical method 397

Oral clearance 391

- health 103, 484

- - education 110

- retention 391

- streptococci 17

Otitis media 374 
pH 83

- cycling 50

Plaque 142

- composition 462

- fluid 278

- pH 455

Polarized light microscopy 43

Preschool children 103, 245

- -, caries disease 155

Prevention 374, 430

- of caries 110

Primary dentition 155

- teeth 25, 345, 354, 405, 442

Probiotic bacteria 374

Profilometry 269, 422

Proteolytic activity 92

Radiation caries 43

Radiation-related caries 43

Radiograph 436

Radiographic phantoms, calibration 314

Radiotherapy 43

Randomized clinical trial 484

- controlled trial 430
Relative income 294

Remineralization 57, 70, 302

Repeatability 397

Reproducibility 397

Root caries 397

Saliva 171, 387, 391

Salivary proteins 163

Salt fluoridation 250

Scanning electron microscopy 43, 269

Secondary caries 97

Sense of coherence 103

Social class 103

Sodium fluoride 126

Solid titration 254, 322

Solubility 322

Sorbitol 331

Stannous fluoride 126, 449

Starch 17

Streptococcus mutans 25, 171, 334

Study designs 331

Sugar alcohols 331

Surface microhardness 163
Survey 245

Synchrotron microcomputed tomography 359

Tin 415

Titanium tetrafluoride 126

Tooth brushing 25, 64

- eruption 491

- wear index 119

- -, prevalence 119

Trimetaphosphate 50

Vanadium chloroperoxidase 334

Visual examination 405

- inspection 36

Water rinsing 64

Xylitol 17, 331, 455

12-year-old children 468

Zero-inflated regression models 468 